\title{
Anesthetic considerations in the management of mucormycosis
}

Mucormycosis or zygomycosis is a well described but a complex disease which appears unseemingly benign but is fatally malignant in its behaviour. Mucormycosis causes severe tissue destruction by endothelial invasion. Our pateint was 65 year old lady with palatal mucormycosis with uncotrolled diabetes who was treated successfully with systemic amphotericin B and surgical debridement which is the main stay of treatment of mucormycosis. Systemic amphotericin B has a lots of side effects like fever, shivering, hypotension, hypoxia, arrhythmia and mainly nephrotoxicity. Our aim is to highlight the need for early diagnosis and surgical debridement of necrotic tissues while keeping in mind the toxic effects of systemic amphotericin $B$ and its interaction with anaesthetic agents.

Key words: Mucormycosis, nephrotoxicity, systemic amphotericin B

\section{INTRODUCTION}

Mucormycosis is a fulminant, opportunistic fungal infection most commonly seen in diabetics and immunocompromised individuals. It is a highly lethal, locally invasive with propensity to involve multiple organs. The six accepted clinical types of mucormycosis are: Rhinocerebral, gastrointestinal, pulmonary and disseminated, burn wound, central nervous system, endocarditis, and vascular mucormycosis.

Prasad K. Kulkarni, Narasimha B. Reddy, B. Shrinivas ${ }^{1}$, Vinita V. Takkalki ${ }^{1}$

Departments of Anaesthesia and ${ }^{1}$ Anaesthesia and Critical Care, MVJ Medical College and Research Hospital, Hoskote, Bengaluru, Karnataka, India

Address for the Correspondence: Dr. Vinita V. Takkalki, MVJ Medical College and Research Hospital, Hoskote, Bengaluru - 562 114, Karnataka, India.

E-mail:vinitavt@gmail.com

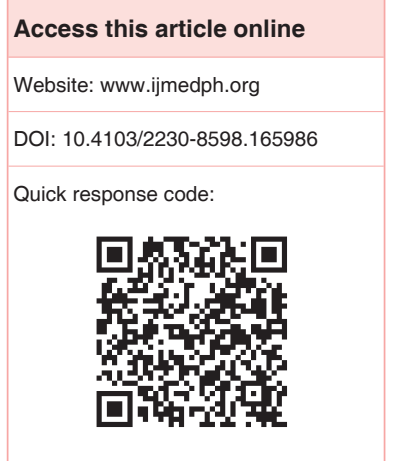

Mortality is high, death can occur within several days to few weeks, in spite of appropriate treatment being administered. Successful management of mucormycosis largely depends on early diagnosis, a reversal of underlying predisposing factors, prompt, and broad surgical debridement of infected tissue and rapid administration of systemic antifungal therapy. There are no established regimes for the primary treatment of mucormycosis.

\section{CASE REPORT}

A 65-year-old diabetic female came with symptoms of systemic infection with palatal perforation secondary to mucormycosis. She had the complaints of high-grade fever with chills since 10 days, severe right sided progressive headache, pain in right eye with swelling of right half of face since 10 days, vomiting (3-4 episodes) with abdominal pain since 1-day, 1 episode of epistaxis, and bleed from oral cavity.

The patient was treated with injection amphotericin B (AmB) deoxycholate in NS at $1 \mathrm{mg} / \mathrm{kg} / \mathrm{day}$ continuous infusion with escalating dose regimen also to other systemic antibiotics. Intravenous AmB has serious adverse effects that include nephrotoxicity, hypotension, arrhythmias, fever, and chills. The patient underwent intraoral anterior maxillectomy and aggressive surgical debridement under general anesthesia. The intraoperatively patient developed premature ventricular complexes with hypotension

This is an open access article distributed under the terms of the Creative Commons AttributionNonCommercial-ShareAlike 3.0 License, which allows others to remix, tweak, and build upon the work non-commercially, as long as the author is credited and the new creations are licensed under the identical terms.

For reprints contact: reprints@medknow.com

How to cite this article: Kulkarni PK, Reddy NB, Shrinivas B, Takkalki VV. Anesthetic considerations in the management of mucormycosis. Int J Med Public Health 2015;5:387-90. 
that were managed with lidocaine infusion and withholding of AmB. Postoperative period was uneventful.

\section{CONCLUSIONS}

Early diagnosis, intravenous AmB, surgical debridement along with treating underlying disease leads to better prognosis in systemic mucormycosis, however attending anesthesiologists should have knowledge of intravenous $\mathrm{AmB}$ induced renal and C.V.S. changes and its interactions.

A 65-year-old female weighing $60 \mathrm{~kg}$ presented to the outpatient clinic with the complaints of high-grade fever with chills since 10 days, severe right sided progressive headache, pain in right eye with swelling of right half of face since 10 days, vomiting (3-4 episodes) with abdominal pain since 1-day, 1 episode of epistaxis, and bleed from oral cavity. She presented with palatal perforation of 6 months duration secondary to mucormycosis with the palatal obturator in situ was posted for intraoral surgical debridement. She is a known case of diabetes mellitus and hypertension since 10 years currently on treatment with regular insulin 20-10-5 IU, tablet telmisartan $40 \mathrm{mg}$ and hydralazine $12.5 \mathrm{mg}$. She had dental extraction 6 months ago.

Preanesthetic evaluation revealed a conscious oriented patient with mild pallor with a heart rate of $120 / \mathrm{min}$ and blood pressure (BP) of 150/90 mmHg. Edema of the right periorbital and maxillary areas, right nostril up to the angle of the mouth on the right side was noted. Maggots were visible in the nasal cavity. Oral examination revealed halitosis, caries tooth, with palatal perforation at the junction of hard and soft palate, which was covered with a removable prosthesis [Figure 1]. Thyromental distance was $6.5 \mathrm{~cm}$; upper lip bite test was grade 2 with a normal temporomandibular joint. Her fasting blood sugar was $245 \mathrm{mg} / \mathrm{dL}$, postprandial blood sugar of $330 \mathrm{mg}$ / $\mathrm{dL}$, and erythrocyte sedimentation rate was $117 \mathrm{~mm} / \mathrm{h}$ with mild microcytic hypochromic anemia. Renal and liver functions tests and serum electrolytes that is, $\mathrm{Na}^{+} 129, \mathrm{~K}^{+} 4.9$ were normal. The rest of the systemic examination was normal. Computed tomographypetri nets revealed features suggestive of chronic maxillary and sphenoidal sinusitis with multifocal erosive lesions with oroantral

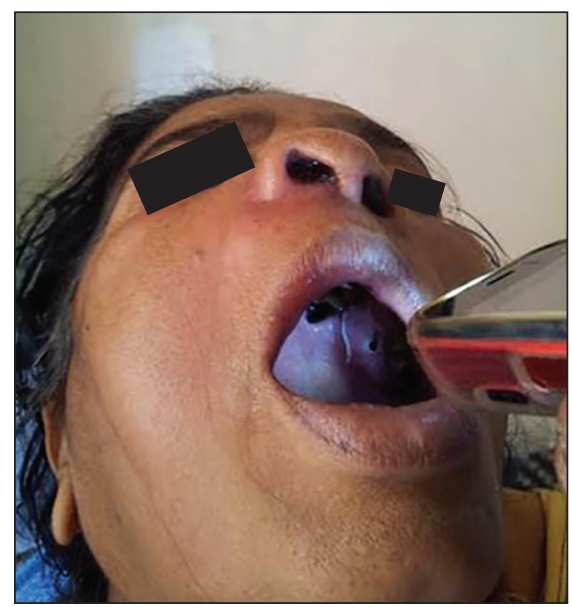

Figure 1: Palatal perforation at the junction of hard and soft palate and oronasal fistula [Figure 2]. She was treated with antibiotics and anti-inflammatory drugs. Blood glucose was controlled with regular insulin. Maggots were cleared with turpentine oil. The patient was started on $\mathrm{AmB}$ deoxycholate after hydrating with $1 \mathrm{~L}$ of NS, followed by dilution of AmB in NS at $1 \mathrm{mg} / \mathrm{kg} /$ day over continuous infusion with titrating the dose. Patient's renal parameters were followed up every $3^{\text {rd }}$ day, and there was an elevation of serum creatinine to $1.7 \mathrm{mg} / \mathrm{dL}$ and serum urea to $34 \mathrm{mg} / \mathrm{dL}$ after 1 -week. The dose of $\mathrm{AmB}$ was reduced to half the dose $(0.5 \mathrm{mg} / \mathrm{kg} /$ day). After 2 days, renal parameters were repeated, which revealed serum creatinine about $1.2 \mathrm{mg} / \mathrm{dL}$ and serum urea $27 \mathrm{mg} / \mathrm{dL}$. Following this patient was taken up for intraoral aggressive surgical debridement under general anesthesia with American Society of Anesthesiology III risk stratification. After obtaining appropriate risk consent and accessing intravenous line, electrocardiography (ECG), pulse oximetry, noninvasive BP (NIBP) monitors were connected. A difficult intubation cart was kept ready. Patient was premedicated with injection glycopyrrolate $0.2 \mathrm{mg}$, injection morphine $10 \mathrm{mg}$, intravenously. After preoxygenation with $100 \%$ oxygen patient was induced with injection thiopentone sodium $2.5 \% 250 \mathrm{mg}$ and palatal perforation was covered with gauze and intubated with $7.5 \mathrm{~mm}$ cuffed reinforced endotracheal tube after relaxation with injection scoline $2 \mathrm{mg} / \mathrm{kg}$ body weight, injection xylocard $1.5 \mathrm{mg} /$ $\mathrm{kg}$ was used to attenuate laryngoscopic reflex. Postintubation pulse oximetry, ECG, NIBP, ETCO ${ }_{2}$, temperature, urine output and neuromuscular monitoring was done. Postintubation BP recording showed a BP of $190 / 100 \mathrm{mmHg}$ with a heart rate of $140 / \mathrm{min}$. The patient was maintained with oxygen, nitrous and isoflurane $1 \%$ with controlled ventilation. Muscle relaxation was maintained with injection vecuronium $0.1 \mathrm{mg} / \mathrm{kg}$ every $30 \mathrm{~min}$. After induction patient developed ventricular ectopics more than $8 / \mathrm{min}$ with BP reading of $92 / 70 \mathrm{mmHg}$ which was treated using ventilation with $100 \% \mathrm{O}_{2}$, injection lidocaine $1.5 \mathrm{mg} / \mathrm{kg}$ bolus dose with $0.5 \mathrm{mg} /$ $\mathrm{kg}$ of infusion over $20 \mathrm{~min}$ and withdrawing the $\mathrm{AmB}$ infusion. Ectopics reduced to $<2 / \mathrm{min}$ with a BP of 106/80 $\mathrm{mmHg}$. After $2 \mathrm{~h}$ the heart rate dropped to $45 / \mathrm{min}$ which was treated with injection

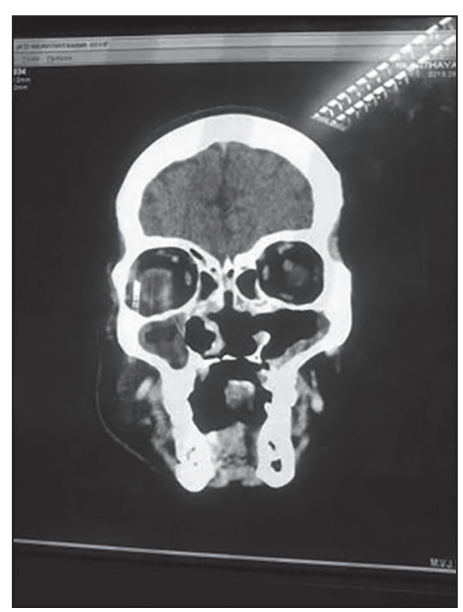

Figure 2: Computed tomography-petri nets revealed features suggestive of chronic maxillary and sphenoidal sinusitis with multifocal erosive lesions with oroantral and oronasal fistula 
atropine $0.6 \mathrm{mg}$. On completion of surgery, she was reversed with injection glycopyrrolate $0.5 \mathrm{mg}$ and injection neostigmine $2.5 \mathrm{mg}$. Patient was extubated and shifted to recovery with $100 \%$ oxygen with $2 \mathrm{~L} / \mathrm{min}$. The rest of the post-operative period was uneventful [Figure 3]. Postoperative echocardiography, renal functions and electrolytes were normal. PAS staining (histopathological report) showed thick walled, ribbon-like, and aseptate hyphae branching at right angles. Therefore, based on the clinical, histopathological, and imaging techniques mucormycosis was revealed as the cause of palatal necrosis. Postoperatively, AmB was continued for a duration of 1 -month while monitoring the renal parameters.

\section{DISCUSSION}

Mucormycosis is an opportunistic fungal infection caused by zygomycetes with a mortality rate of $35-96 \%$ depending upon the site and extent of the disease. ${ }^{[1]}$

The classical features of mucormycosis are angio-invasion, thrombosis, infarction and necrosis. Our patient had long-standing diabetes mellitus and making her immunologically compromised with dental extraction providing probable entry port.

Treatment includes extensive surgical debridement, high doses of systemic AmB, control of underlying disease and other supportive measures. ${ }^{[1]}$ Despite advances in the development of new agents, $\mathrm{AmB}$ remains a cornerstone of antifungal therapy for patients with opportunistic fungal infections. Antifungal drugs have poor penetration ability at the site of infection. Traditionally, $\mathrm{AmB}$ is given in 2-6 $\mathrm{h}$ infusions on the basis of the assumption that the severity and frequency of related side effects increase during more rapid administration. Recently, continuous infusion of $\mathrm{AmB}$ has been shown to reduce the incidence of nephrotoxicity and infusionrelated side effects. Accordingly, our patient was given a continuous infusion of $\mathrm{AmB}$ at a dose of $1 \mathrm{mg} / \mathrm{kg}$ body weight to be titrated and escalated on 3 days interval. ${ }^{[2]}$

Hypokalemia, hypomagnesemia, fever, chills, dyspnea, and hypotension are common side effects of AmB. Allergic reactions, seizures, anemia, and thrombocytopenia are less likely to occur but are well-documented. Renal function is impaired in $>80 \%$ of treated patients, and a permanent decrease in the glomerular filtration rate is likely, with $15 \%$ of patients requiring hemodialysis. Studies indicate that the anesthetic management of patients with acute tubular necrosis leading to the development of acute renal failure is

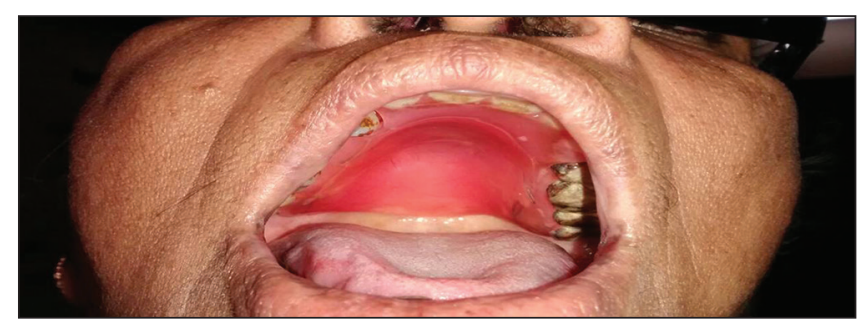

Figure 3: Postoperative image of healed palatal perforation with obturator of particular concern for anesthesia providers. ${ }^{[3]}$ The morbidity and mortality of patients with acute renal failure are so high that only life-saving surgery should be undertaken. ${ }^{[3]}$ Anesthesia providers must pay particular attention for the maintenance of an adequate mean arterial pressure and cardiac output while concomitantly avoiding further renal insults. Our patient had hypotension intraoperatively with arrhythmias that responded to treatment with lidocaine infusion. A heightened awareness for renal, electrolyte, coagulopathic, hemodynamic, and respiratory aberrancies is warranted for anesthesia providers when treating patients receiving AmB therapy. ${ }^{[3]}$

AmB acts by binding to the sterol component, ergosterol, of the cell membrane of susceptible fungi forming transmembrane channels leading to alterations in cell permeability through which monovalent ions $\left(\mathrm{Na}^{+}, \mathrm{K}^{+}, \mathrm{H}^{+}\right.$, and $\left.\mathrm{Cl}^{-}\right)$leak out of the cell resulting in cell death. It can also bind to the cholesterol component of the mammalian cell leading to cytotoxicity.

The metabolic pathways of $\mathrm{AmB}$ after administration have not been defined.

Concurrent use of corticosteroids, ACTH and digitalis glycosides may potentiate hypokalemia, which could predispose the patient to cardiac dysfunction and digitalis toxicity. If used concomitantly, serum electrolytes and cardiac function should be closely monitored. AmB-induced hypokalemia may enhance the effect of skeletal muscle relaxants. When administered concomitantly, serum potassium levels should be closely monitored. Arrhythmia, atrial fibrillation, bradycardia, cardiac arrest, cardiomegaly, hemorrhage, postural hypotension, and vasodilatation have been reported. The typical dose is $1-1.5 \mathrm{mg} / \mathrm{kg} / \mathrm{d}$. The total dose given over the course of therapy is usually $2.5-3$ g. ${ }^{[2,4,5]}$

Our patient posed us three challenges:

1. Difficult airway in view of palatal perforation with obturator in situ

a. The palatal obturator can cause anesthetic complications including airway obstruction,

b. Inability to pass endotracheal tube due to dislodgement of prosthesis,

c. limitation of the space for a laryngoscope and a traumatic intubation. ${ }^{[6]}$

2. Long standing diabetes mellitus.

3. Continuous infusion of $\mathrm{AmB}$ which could interact with anesthetic agents and produce an adverse outcome.

Hyperbaric oxygen has been used as an adjunct to aggressive surgical debridement, AmB therapy, and control of any underlying predisposing conditions. Although studies have shown that hyperbaric oxygen exerts a fungistatic effect, the most important effect of hyperbaric oxygen is to aid revascularization, with subsequent healing in poorly perfused acidic and hypoxic but viable areas of tissue. ${ }^{[7]}$ 
Interestingly, isoflurane has recently been found to halt fungal growth in vitro and could offer future therapeutic potential for certain systemic fungal infections. ${ }^{[3]}$

\section{Acknowledgment}

Dr. A.V. Pai, Prof. and HOD, Department of Anaesthesiology, MVJ Medical College and Research Hospital.

\section{Financial support and sponsorship}

Nil.

\section{Conflicts of interest}

There are no conflicts of interest.

\section{REFERENCES}

1. Marchetti A, Jayachandran A, Guha A. Post-traumatic invasive mucormycosis. J Intensive Care Soc 2011;12:143-4.

2. Imhof A, Walter RB, Schaffner A. Continuous infusion of escalated doses of amphotericin B deoxycholate: An open-label observational study. Clin Infect Dis 2003;36:943-51.

3. Choleva AJ. Anesthetic management for lobectomy in a patient with coccidioidomycosis: A case report. AANA J 2010;78:321-5.

4. Laniado-Laborín R, Cabrales-Vargas MN. Amphotericin B: Side effects and toxicity. Rev Iberoam Micol 2009;26:223-7.

5. Rowles DM, Fraser SL. Amphotericin B lipid complex (ABLC)-associated hypertension: Case report and review. Clin Infect Dis 1999;29:1564-5.

6. Ahuja V, Kazal S, Sinha S. Palatal obturator: Perioperative concerns. Indian J Anaesth 2014;58:87-9.

7. Dash AK, Arora V, Nagarkar NM. Rhino-orbital mucormycosis. Natl J Otorhinolaryngol Head Neck Surg 2013;10:5-6. 